\title{
EDITORIAL
}

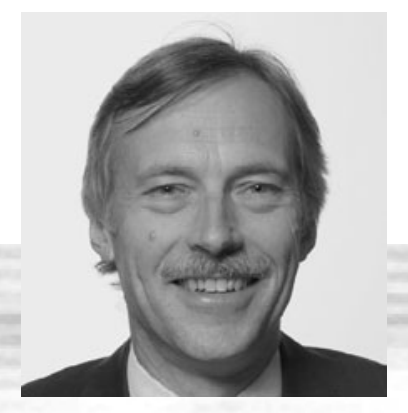

Arndt Bode

Hauptherausgeber

\section{Zuse und Industrialisierung}

Im hundertsten Geburtsjahr von Konrad Zuse ist es angemessen, den Schwerpunkt der wissenschaftlichen Arbeiten der Informatik in der Rückschau zu beobachten. Vor etwa 70 Jahren hat Konrad Zuse seine Gedanken zum programmierbaren Allzweckrechner materialisiert und damit die Grundlage für die atemberaubende Entwicklung der neuen Wissenschaft Informatik geliefert. Lagen die Schwerpunkte der Informatikforschung bis etwa 1980 nahezu gleich verteilt in den Bereichen der Technischen Informatik, die sich mit der Konstruktion des Computers befasst, der Praktischen Informatik, die sich mit seiner Programmierung beschäftigt, der Theoretischen Informatik, die Algorithmen und Strukturen beschreibt und den Anwendungen der Informatik in verschiedensten Fachgebieten, so hat sich in den letzten 30 Jahren das Interesse der Informatikforschung stärker in den Bereich der Anwendungen und der Programmiertechniken verlagert. Die Industrialisierung des Software-Entwurfs und damit die ingenieurmäßige Beherrschung der Nutzung des Computers für die verschiedensten Anwendungen unter klar berechenbaren Randbedingungen hinsichtlich Kosten, Zuverlässigkeit und Vertraulichkeit ist ein wesentliches Anliegen heutiger Informatikforschung. So ist es auch kein Wunder, dass alle Beiträge in einem themenoffenen Heft des Informatik-Spektrums im weitesten Sinne diesem Bereich zuzuordnen sind.

Holger von Jouanne-Diedrich, Juliane Blechinger, Christoph Neumann, Richard Lenz und Stefan Schwarz von der Siemens AG bzw. dem Lehrstuhl für Informatik 6 der Universität Erlangen-Nürnberg berichten in ihrem Hauptbeitrag zur "Integration verteilter und heterogener Configuration-Management-Datenbanken" aus dem Bereich des IT Service Managements zur Verwaltung verteilter heterogener und autonomer Systeme in großen Einrichtungen. Es wird eine Anforderungs- und Marktanalyse mit dem Vergleich verschiedener Produkte der wesentlichen Hersteller gegeben.

"Serviceorientierte Vorgehensmodelle: Überblick, Klassifikation und Vergleich" ist das Thema von Oliver Thomas, Universität Osnabrück und Katrina Leyking und Michael Scheid vom Institut für Wirtschaftsinformatik im Deutschen Forschungszentrum für Künstliche Intelligenz, Saarbrücken. Grundidee der serviceorientierten Architektur (SOA) ist es, Systeme aus eigenständigen Softwarefunktionen zusammenstellen zu können. Die Autoren gehen auf die historische Entwicklung der Bewertung des SOA-Konzepts ein und vergleichen serviceorientierte Vorgehensmodelle.

Im Aktuellen Schlagwort wird von Henrik Grosskreutz, Benedikt Lemmen und Stefan Rüping vom Fraunhofer Institut für Intelligente Analyse- und Informationssysteme in St. Augustin über effiziente Suche in großen Datenmengen unter Berücksichtigung von Privatheitsgesichtspunkten (Privacy-Preserving Data Mining) berichtet.

In der Student's Corner beschreibt Dirk Holz mit dem Beitrag "Effiziente Kartografie und Navigation für mobile Service Roboter" ein erfolgreiches Projekt, das in verschiedenen RoboCup Wettbewerben hervorragende Platzierungen erreichen konnte.

Für diese Beiträge, wie für die üblichen Rubriken des Informatik-Spektrums, wünsche ich Ihnen anregende Lektüre.

\section{Arndt Bode,}

Hauptherausgeber 